\title{
Impacto de la perfusión hipotérmica pulsativa en el injerto renal de donante subóptimo: nuestra experiencia inicial
}

\author{
Estefanía Iglesias-González¹, Joan Torras-Ambros² \\ ${ }^{1}$ Departamento Extracción Multiorgánica y Trasplante Renal. Hospital Universitario de Bellvitge. Hospitalet de \\ Llobregat. Barcelona. España \\ ${ }^{2}$ Departamento de Nefrología. Hospital Universitario de Bellvitge. Hospitalet de Llobregat. Barcelona. España
}

Como citar este artículo: Iglesias-González E, Torras-Ambros J. Impacto de la perfusión hipotérmica pulsativa en el injerto renal de donante subóptimo: nuestra experiencia inicial. Enferm Nefrol. 2020 Ene-Mar;23(1):60-66

\section{Resumen}

Objetivo: Estudios recientes han demostrado que el mantenimiento de la viabilidad de riñones con criterios expandidos durante su preservación sea un reto. La máquina de perfusión hipotérmica pretende mitigar el efecto del almacenamiento en frío sobre la calidad del órgano cuando el tiempo de isquemia fría es prolongada o el donante subóptimo.

Objetivo: Evaluar las complicaciones que presentan los pacientes trasplantados renales con preservación estática fría o perfusión hipotérmica pulsátil.

Material y Método: Estudio observacional retrospectivo durante 2010-2012 donde se incluyeron todos los trasplantes renales realizados en un hospital de tercer nivel. Las variables de estudio: estancia hospitalaria, horas de isquemia, necesidad de diálisis y número de sesiones post trasplante y el dispositivo de almacenamiento, edad y patologías asociadas al donante.

Resultados: Se realizaron 175 trasplantes donde 70 procedieron de donantes $\geq 65$ años. Se perfundieron en máquina 30 riñones y en 40 se utilizó la preservación estática. Nuestros hallazgos respecto al uso de la má-

\section{Correspondencia:}

Estefanía Iglesias González

E-mail: eiglesiasg@bellvitgehospital.cat quina de perfusión conllevan un descenso en la estancia media hospitalaria y una menor necesidad de hemodiálisis postrasplante.

Conclusiones: Debido al alto porcentaje de órganos procedentes de donantes de edad avanzada y difíciles de preservar, resulta fundamental buscar técnicas de perfusión intravascular continua para una preservación más efectiva del órgano.

PALABRAS CLAVE: injerto renal; trasplante renal; perfusión hipotérmica pulsátil.

Impact of hypothermic pulsatile perfusion on the suboptimal kidney donors: our initial experience

\section{Abstract}

Introduction: Recent studies have shown that maintaining kidney viability with expanded criteria during preservation is a challenge. The hypothermic infusion machine aims to mitigate the effect of cold storage on the quality of the organ when the time of cold ischemia is prolonged or the donor is suboptimal.

Objective: To evaluate the complications presented by renal transplanted patients with cold static preservation or hypothermic pulsatile perfusion. 
Material and Method: Retrospective observational study during 2010-2012, including all kidney transplant patients in a third level hospital. The study variables: length of stay, hours of ischemia, need for dialysis and number of post-transplant sessions and the storage device, age and pathologies associated with the donor.

Results: 175 transplants were performed, 70 of which were donors $\geq 65$ years old. 30 kidneys were perfused in a machine and static preservation was used in 40. Our findings regarding the use of the infusion machine lead to a decrease in the average length of stay and a reduced need for post-transplant hemodialysis.

Conclusions: Due to the high percentage of organs difficult to preserve and from elderly donors, it is essential to seek continuous intravascular perfusion techniques for a more effective preservation of the organ.

KEYWORDS: renal graft; renal transplantation; hypothermic pulsatile perfusion.

\section{Introducción}

El aumento progresivo de la esperanza de vida y la prevalencia de enfermedades renales crónicas han generado un mayor número de personas que requieren un trasplante $^{1}$. Sin embargo, existe una escasez de donantes ideales para los trasplantes, siendo necesario adoptar diferentes estrategias para conseguir una mayor oferta de órganos: aumentar la donación de vivo, obtener órganos de donantes en asistolia y con criterios expan$\operatorname{didos}^{2,3}$.

Los riñones de donantes con criterios expandidos (DCE) término, que agrupa a donantes que poseen un riesgo global de rechazo en el receptor de 1,7 veces mayor que los receptores de órganos de donantes ideales, parecen tener una mayor tasa de complicaciones, tales como, una elevada función retardada y fracaso renal derivando a una inferior supervivencia del injerto a largo plazo ${ }^{4,5}$. Cuando un donante renal es mayor de 70 años existe un mayor riesgo de pérdida del injerto que cuando es se menor de 60 años 6 . Sin embargo, la supervivencia de los pacientes con estos riñones es más alta que los que permanecen en diálisis ${ }^{7}$, beneficio que se observa incluso, en los ancianos que reciben riñones de donantes con criterios expandidos ${ }^{8-10}$.
En España, desde 2011, más de la mitad de los donantes tienen más de 60 años, cerca de un tercio más de 70 y casi un $10 \%$ más de 80 , con un máximo histórico registrado de un donante de riñón de $90 \mathrm{años}^{11}$. EI trasplante renal procedente de DCE presenta una tasa superior de función retardada incrementando los costes postoperatorios a expensas de prolongación de estancia hospitalaria, necesidad de diálisis y procedimientos de diagnóstico adicionales. La máquina de perfusión pretende mitigar el efecto del almacenamiento en frío sobre la calidad del órgano cuando el tiempo de isquemia fría es prolongado o el donante es subóptimo. La perfusión hipotérmica puede ser superior a la preservación estática fría al reducir el riesgo de función retardada, disfunción primaria y aumento de la supervivencia al año del injerto renal. El mantenimiento de la viabilidad de los órganos durante la preservación se convierte en un reto, parece apropiado buscar estrategias como la perfusión pulsátil para optimizar el trasplante de riñones longevos y mejorar resultados. El futuro puede ser la perfusión en máquina, por este motivo, nuestro centro impulsó un nuevo proyecto basado en la preservación renal pulsativa, con el objetivo de lograr una perfusión intravascular continua con una preservación más efectiva.

\section{Objetivos}

El objetivo principal del presente estudio fue evaluar las complicaciones que presentan los pacientes trasplantados renales con dos tipos de preservación diferentes: preservación estática fría o perfusión hipotérmica pulsátil.

\section{Metodología}

Se realizó un estudio de tipo cohortes retrospectivo en un hospital de tercer nivel, desde febrero de 2010 hasta enero de 2012. La muestra del estudio fue todo paciente trasplantado renal que recibió un riñón procedente de donante fallecido en muerte encefálica con criterios expandidos, analizándose, la evolución a corto plazo de los receptores de injertos renales procedentes de donantes $\geq 65$ años. Los sujetos de la muestra fueron divididos en dos grupos según el método de preservación renal utilizado pre trasplante: conservación estática en frío (CS) o máquina de perfusión hipotérmica pulsativa (MP). Los criterios expandidos se definieron de acuerdo con la United Network of Organ Sharing 
(UNOS) ${ }^{12,13}$ que incluye: la edad del donante $\geq 60$ años 0 50-60 años con al menos dos de los siguientes aspectos: antecedentes de hipertensión, cerebrovasculares, causa de la muerte y creatinina en suero $\geq 132 \mathrm{~mol} / \mathrm{l}(1,5 \mathrm{mg} /$ dl) antes de la recuperación de órganos.

Se incluyeron todos los órganos renales generados, tanto intra como extrahospitalarios, durante el periodo de estudio procedente de donante por muerte encefálica y $\geq 65$ años en el momento de la donación orgánica. Se acordó este rango de edad porque un donante ya es considerado con criterios expandidos sin tener que valorar la presencia de otros criterios.

Se excluyeron todos los riñones procedentes de donantes ideales ( $<50$ años y sin patología asociada) por no estar indicada la utilización de la máquina de perfusión pulsativa en la preservación renal, así como, los destinados para el trasplante de órganos combinado (por ejemplo: hepato-renal).

Las principales variables de estudio fueron: tiempo de estancia hospitalaria, horas de isquemia fría, necesidad de diálisis y número de sesiones post trasplante y el dispositivo de preservación empleado. Además, se recogieron variables tanto del donante (sociodemográficas, clínicas y variables pre-trasplante: edad, género y comorbilidades asociadas del donante) como de los receptores renales, para analizar de qué manera se interrelacionaban entre ellas. En el receptor se analizaron directamente aquellos datos que nos dieron una visión general de cual fue la evolución de los riñones trasplantados.

Todos los riñones fueron recuperados y trasplantados por nuestro equipo de extracción y trasplante, esto evitó el sesgo de los resultados debido a las diferentes habilidades quirúrgicas. Cuando fue posible, de cada donante, un riñón fue asignado a MP y el contralateral a CS. Cuando uno de los dos riñones no era adecuado para el trasplante en nuestro hospital, el riñón fue asignado a MP o CS basado en la disponibilidad de la máquina y desechables MP. Cuando uno de los dos riñones se remitió para su trasplante a otro hospital debido a la política local de intercambio de órganos, nuestro riñón se asignó al azar a MP o CS. La adquisición de la técnica fue la misma en los grupos CS y MP.

El método de preservación se estableció en el momento de la aceptación del riñón para trasplante. Todos riñones fueron sometidos a lavado vascular in situ con solución de preservación fría durante la extracción orgánica. Riñones preservados en MP se conectaron inmediata- mente después de la extracción hasta su trasplante y los riñones preservados en CS se almacenaron en un recipiente con solución de preservación fría de acuerdo con la rutina establecida en el centro. Los riñones se evaluaron individualmente antes del trasplante basándonos en las características de los donantes y los hallazgos histológicos en el CS grupo y en las variables anteriores además de los parámetros de la máquina en el grupo MP. Nuestros patólogos realizaron una evaluación basada en una biopsia pre-trasplante, que se realizó en todos los casos y la puntuación se utilizó para evaluar la viabilidad del riñón. En todo momento se respetó el anonimato y el tratamiento correcto de los datos obtenidos.

Los datos fueron recogidos en una base de datos estandarizada y el análisis estadístico se realizó utilizando PASW Statistics 13.0 (SPSS Inc., Chicago, IL, EE.UU.). Para el análisis comparativo de los grupos se utilizó el test de la $t$ de Student, para comparar dos variables continuas y la prueba no paramétrica de Mann-Whitney cuando no se consideró aceptable utilizar la distribución de la propiedad normal. Los datos se expresaron como media y desviación estándar en todas las variables un valor de $p$ de 0,05 o inferior fue considerado estadísticamente significativo.

Se solicitaron los permisos necesarios para la obtención de los datos al centro hospitalario y el estudio fue aprobado por el Comité Ético de Investigación Clínica de dicho centro. El anonimato y el tratamiento correcto de los datos obtenidos se respetaron en todo momento de acuerdo con las pautas aprobadas por el comité de ética e investigación del centro.

\section{Resultados}

En el periodo analizado se realizaron un total de 175 trasplantes, de los cuales, fueron incluidos para el estudio 70 pacientes $(40 \%)$ procedentes de la lista de espera del centro hospitalario que recibieron un riñón procedente de un donante $\geq 65$ años. Estos 70 pacientes fueron divididos en dos grupos en función del método de preservación renal utilizado ya fuera máquina de perfusión pulsativa o preservación estática fría. Un total de 30 pacientes $(42,45 \%)$ fueron trasplantados con un injerto renal preservado en máquina de perfusión pulsativa y a 40 pacientes $(57,14 \%$ ) se les implantó un riñón procedente de preservación estática. La edad media del donante cuyos riñones fueron preservados en maquina fue de 73,27 años y de 75,6 años en CS, siendo superior en este último grupo. 
En el grupo de los donantes cuyos órganos renales se preservaron en MP un $90 \%$ de los casos $(n=27)$ la causa de la muerte encefálica fue por un accidente cerebro vascular (ACV) y presentaron una tasa de diabetes mellitus del $23,33 \%(n=7)$ y un $73,33 \%(n=22)$ de hipertensión arterial (HTA). En el grupo los donantes cuyos órganos renales se preservaron en CS un $77,50 \%(n=31)$ sufrió un AVC con una tasa de diabetes mellitus del $22,5 \%(n=9)$ e HTA del $50 \%(n=20)$. El tiempo medio de isquemia fría previa al trasplante fue ligeramente mayor en los riñones preservados en CS siendo de 20,13 horas por las 19,30 horas para el grupo de MP.

La terapia de inducción e inmunosupresor crónico fue el mismo para ambos grupos de acuerdo con los protocolos hospitalarios. Los parámetros que caracterizan al riñón durante la perfusión, como resistencia vascular, flujo y marcadores de viabilidad no se utilizaron para el análisis.

La tasa de necrosis tubular aguda (NTA), definida como necesidad de hemodiálisis postrasplante, fue del $20 \%$ en MP y del $35 \%$ en CS. La media de sesiones de hemodiálisis postrasplante fue de 1,07 en MP y 2,58 en CS y la estancia hospitalaria media fue de 14,89 y 19,33 días para los grupos de MP y CS respectivamente.

Los resultados obtenidos reflejan que, a igualdad de tiempo de isquemia fría, el uso de la MP durante la preservación renal se asociaba a una menor presencia de NTA, a una menor necesidad de hemodiálisis postrasplante y a un descenso en la estancia media hospitalaria. Realizado el análisis de las variables mostró que con el uso de la MP hubo una tendencia hacia una mejor función del injerto postrasplante inmediato que pudo reflejarse por el menor número de sesiones de diálisis necesarios con la consiguiente disminución de la estancia hospitalaria para el grupo MP (Tabla 1), lo cual, fue confirmado por medio de regresión logística $(95 \%$ de confianza $p=0,131)$, sin embargo, estadísticamente no se encontró diferencia significativa.

\section{Discusión}

El trasplante es uno de los grandes avances en la medicina, no sólo por el papel en la recuperación de la salud sino también por la mejoría de la calidad de vida en personas con enfermedades crónicas o terminales por falla de algún órgano ${ }^{14}$.

En España más del $50 \%$ de los donantes son mayores de 60 años. En nuestro estudio, el $40 \%$ se sitúan entre los 50 y 70 años y prácticamente todos tienen algún antecedente patológico o factor de riesgo cardiovascular. Este aumento de edad conlleva que más de la mitad de los riñones generados provengan de donantes subóptimos. Estos órganos menos adecuados son más difíciles de preservar y son necesarios nuevos métodos de preservación.

Asegurar una preservación renal exitosa entre la extracción del órgano y su implantación es de reconocida importancia desde los inicios del trasplante renal. Con el paso del tiempo se han desarrollado diferentes enfoques en CS y MP para limitar el daño isquémico evolucionando ambas técnicas desde su desarrollo temprano ${ }^{15}$.

La perfusión continua hipotérmica fue descrita por Humphries (1967) y desarrollada por Belzer un año más tarde ${ }^{16}$ pero su complejidad y alto coste inicial minimizaron su uso. No obstante, en las dos últimas décadas ha habido un renovado interés basado en una mejor preservación renal de donantes a corazón parado y con criterios expandidos.

Estudios recientes, sugieren que la máquina de perfusión hipotérmica podría tener un beneficio clínico, en términos de supervivencia del injerto, durante el primer

Tabla 1. Variables postrasplante según grupo.

\begin{tabular}{lccc}
\hline Variable & Máquina Perfusión hipotérmica & $\begin{array}{c}\text { Preservación hipotérmica } \\
\text { Media } \pm \text { Desviación estándar }\end{array}$ & Valor de $\mathbf{p}$ \\
\hline Tiempo estancia hospitalaria (días) & $14,89 \pm 11,69$ & $19,33 \pm 19,66$ & 0,241 \\
\hline Tiempo de isquemia (horas) & $19,3 \pm 3,64$ & $20,13 \pm 4,20$ & 0,392 \\
Sesiones de hemodiálisis (número) & $1,07 \pm 2,46$ & $2,58 \pm 5,54$ & 0,131 \\
\hline
\end{tabular}


año postrasplante y una menor tasa de función renal retardada en comparación con el almacenamiento en frío ${ }^{17}$. Según el Informe de la United Network for Organ Sharing (2003), conllevó un menor retraso en la recuperación de la función glomerular y una mejor función inmediata del injerto que la conservación en fríio ${ }^{18}$. En sus publicaciones, Moers (2009) afirmó que la preservación con máquina de perfusión hipotérmica se asoció a un descenso del riesgo de desarrollar función retardada y mejoraba la supervivencia del injerto en el primer año postrasplante ${ }^{17}$.

Los cambios isquémicos comienzan con la muerte cerebral debido a inestabilidades hemodinámicas y a la isquemia fría antes del trasplante que se suma a la lesión isquémica renal. La MP juega un papel importante en la modulación de la lesión por reperfusión isquémica a consecuencia de la rápida liberación de radicales libres poco después de la reperfusión.

La MP facilita el almacenamiento durante periodos más largos ${ }^{19}$ aunque es nuestro estudio este aspecto no fue valorable puesto que los tiempos de isquemia fría fueron semejantes. También permite la evaluación de la extensión del daño isquémico del órgano e incluso mejora la evaluación de la calidad de los riñones antes del trasplante que, junto con parámetros de los donantes, aporta una predicción de la función renal postrasplan$\mathrm{te}^{20}$. La preservación renal de injertos de DCE mediante MP además de aportar información adicional sobre la viabilidad del injerto, reduce la incidencia de rechazo crónico y de $\mathrm{NTA}^{21}$, enfoques que parecen reflejarse en el presente estudio. Asimismo, se encontró una reducción en la necesidad de sesiones de diálisis cuando se produjo una función retardada del injerto (FRI) con una reducción de la estancia media hospitalaria.

Es bien conocido que una reducción en la incidencia de FRI conlleva a una reducción en la necesidad para diálisis después de la intervención. La menor necesidad de diálisis está probablemente relacionada con la menor gravedad de la FRI para aquellos pacientes que recibieron un riñón conservada por MP22.

Los costes económicos son uno de los principales inconvenientes a la tecnología de MP. Wszola (2009) y Groen (2012), Ilegaron a la conclusión que con el uso de la perfusión en máquina había un menor coste a largo plazo y una amortización de la inversión realizada a los 16 meses postrasplante ${ }^{23,24}$. Varios estudios recientes confirman el beneficio económico de MP cuando se utiliza como método de conservación preferente ${ }^{25,26}$. La MP reduce el riesgo de FRI y fracaso del injerto con costes más bajos que CS, junto con el aumento de los años de vida y los años de vida ajustados por calidad, al mismo tiempo ${ }^{27}$.

En cuanto a las limitaciones del estudio, son las derivadas del diseño, ya que se trata de un estudio descriptivo retrospectivo. En segundo lugar, no se puede contrastar la calidad de la recogida de datos, puesto que, en su momento, cuando se registraron, no derivaba de un diseño de investigación sino de una actividad clínica rutinaria.

En conclusión, nuestros resultados sugieren que los injertos procedentes de un DCE presentan una mayor susceptibilidad al daño isquémico, una mayor tasa de función renal retardada y en términos generales peores resultados a medio y largo plazo. La MP mejora el resultado del trasplante, es una herramienta eficiente que reduce la incidencia y la severidad de la FRI aumentando la supervivencia del injerto de los pacientes trasplantados con riñones de DCE. El papel del método de conservación parece ser crucial para el resultado del trasplante renal, especialmente en DCE, enfoque que parece reflejarse en nuestro estudio.

Recibido: 04-01-19

Revisado: 15-02-19

Modificado: 25-11-19

Aceptado: 15-12-19

\section{Bibliografía}

1. García-García G, Harden P, Chapman J. The global role of kidney transplantation. Nefrologia 2012;32(1):1-6.

2. Moers C, Kornmann NS, Leuvenink HG, Ploeg RJ. The influence of deceased donor age and old-for-old allocation on kidney transplant outcome. Transplantation 2009;88(4):542-52.

3. Saidi RF, Elias $N$, Kawai T, Hertl M, Farrell ML, Goes $N$, et al. Outcome of kidney transplantation 
using expanded criteria donors and donation after cardiac death. Am J Transplant 2007;7:2769-74.

4. Audard V, Matignon M, Dahan K, Lang P, Grimbert $P$. Renal transplantation from extended criteria cadaveric donors: problems and perspectives overview. Transpl Int 2008;21:11-7.

5. Wynn J, Alexander C. Increasing organ donation and transplantation: the U.S. experience over the past decade. Transpl Int 2011;24:324-32.

6. Valeri M, Torlone $N$, Meçule A. Five years experience in a senior kidney donor programme. Cells Tissues Organs 2006;2:99-102.

7. 0jo A0, Hanson JA, Meier-Kriesche $H$, Okechukwu $C N$, Wolfe RA, Leichtman $A B$, et al. Survival in recipients of marginal cadaveric donor kidneys compared with other recipients and wait-listed transplant candidates. J Am Soc Nephrol 2001;12:589-97.

8. Rao PS, Merion RM, Ashby VB, Port FK, Wolfe RA, Kayler LK. Renal transplantation in elderly patients older than 70 years of age: results from the Scientific Registry of Transplant Recipients. Transplantation 2007;83(8):1069-74.

9. Wolfe RA, Ashby VB, Milford EL, 0jo A0, Ettenger RE, Agodoa LY, et al. Comparison of mortality in all patients on dialysis, patients on dialysis awaiting transplantation, and recipients of a first cadaveric transplant. N Engl J Med 1999;341(23):1725-30.

10. Escalante JL, Río F. Preservación de órganos. Med Intensiva 2009;33(6):282-92.

11. Organización Nacional de Trasplantes. Memoria actividad donación y trasplante renal. España 2018. [Internet] [Consultado 2 mar 2019]. Disponible en: http://www.ont.es/infesp/Memorias/Actividad\%20de\%20Donaci\%C3\%B3n\%20y\%20 Trasplante\%20Renal.pdf.

12. Treckmann J, Moers C, Smits JM, Gallinat A, Maathuis $\mathrm{MH}$, Van Kasterop-Kutz M, et al. Machine perfusion versus cold storage for preservation of kidneys from expanded criteria donors after brain death. Transpl Int 2011;24:548.
13. Metzger RA, Delmonico FL, Feng $S$, Port FK, Wynn JJ, Merion RM. Expanded criteria donors for kidney transplantation. Am J Transplant 2003;3(4):114-25.

14. Groen H, Moers C, Smits JM, Treckmann J, Monbaliu D, Rahmel A, et al. Cost-effectiveness of hypothermic machine preservation versus static cold storage in renal transplantation. Am J Transplant 2012;12:1824-30.

15. Choy YW, Bunnapradist S, Choe ES, Stadtler M, Simmons V, Locke J, et al. Can machine perfusion decrease the likelihood of discard among biopsied kidneys? Transplant Proc 2008;40:1029-31.

16. Moers C, Smits JM, Maathuis MH, Treckmann J, Van Gelder F, Napieralski BP, et al. Machine perfusion or cold storage in deceased-donor kidney transplantation. N Engl J Med 2009;360:7-19.

17. Wight JP, Chilcott JB, Holmes MW, Brewer N. Pulsatile machine perfusion vs cold storage of kidneys for transplantation: a rapid systematic review. Clin Transplant 2003;17:293-307.

18. Wszola M, Kwiatkowski A, Latek M, Ostrowski K, Domagala $P$, Ciszek $M$, et al. Long term medical and economical benefit of machine perfusion storage in comparison to cold storage. Ann Transplant 2009;14(2):24-9.

19. Matsuoka I, Almeda J, Mateo R. Pulsatile perfusion of kidney allografts. Curr Opin Organ Transplant 2009;14(4):365-69.

20. Domagala $P$, Kwiatkowski A, Perkowskaa-Ptasinska A, Wszola M, Panufnik $L$, Paczek $L$, et al. Assessment of kidneys procured from expanded criteria donors before transplantation. Transplant Proc 2009;41:2966-69.

21. Kwiatkowski $A$, Wszoła $M$, Perkowska-PtasinsKa A, Ostrowski K, Domagała P, Fesołowicz S, et al. Influence of preservation method on histopathological lesions of kidney allografts. Ann Transplant 2009;14:10-3.

22. Wight JP, Chilcott JB, Holmes MW, Brewer N. Pulsatile machine perfusion vs cold storage of kidneys for transplantation: a rapid and systematic review. Clin Transplant 2003;17:293-307. 
23. Groen H, Moers C, Smits JM, Treckmann J, Monbaliu D, Rahmel $A$, et al. Long Term Cost-Effectiveness of Hypothermic Machine Perfusion Versus Static Cold Storage in Kidney Transplantation. Am J Transplant 2012;12(7):1824-30.

24. Ureña A, Fernández 0, Arango A, Rebollo P. Calidad de vida en trasplante renal. En: Libro de comunicaciones del XXI Congreso de la Sociedad Española de Enfermería Nefrológica. Barcelona: HOSPAL; 1996.

25. Garfield S, Evans R. Machine perfusion cost-effectiveness versus cold storage has been demonstrated; limiting use to marginal donor kidneys unjustified. Transpl Int 2010;23:67-8.
26. Lenihan $C, 0^{\prime}$ Kelly $P$, Mohan $P$, Little $D$, Walshe JJ, Kieran NE, et al. MDRD-estimated GFR at one year post-renal transplant is a predictor of long-term graft function. Ren Fail 2008;30:345-52.

27. Stratta RJ, Rohr MS, Sundberg AK, Armstrong G, Hairston G, Hartmann $E$, et al. Increased kidney transplantation utilizing expanded criteria deceased organ donors with results comparable to standard criteria donor transplant. Ann Surg 2004;239:68895.

Este artículo se distribuye bajo una Licencia Creative Commons Atribución-NoComercial 4.0 Internacional. https://creativecommons.org/licenses/by-nc/4.0/

Open Access (c) (7) (8) 